\title{
Covariant Perturbation Theory of Non-Abelian Kinetic Theory *
}

\author{
Zheng Xiaoping ${ }^{2}$ Li Jiarong $^{1}$ \\ 1 The Institute of Particle Physics, Huazhong Normal University,Wuhan,P.R.China \\ 2 Department of Physics,Zhongshan University,Guangzhou,P.R.China
}

September 6, 2018

\begin{abstract}
A double perturbation idea is presented in framework of the quark-gluon plasma kinetic theory. A solvable set of equations from the 'double perturbation' is derived and the equations are showed to be gauge-independent. The formalism of Landau damping rate for the plasmon at zero momentum is given and discussed.
\end{abstract}

PACS number: 12.38.Mh

In recent ten years, much interest is focused on the study of non-Abelian plasma from transport theory 11. It is well-known that kinetic theory is believed to describe correctly the quark-gluon plasma(QGP) physics as well as temperature field theory[3] and be extended to out-of-equilibrium situations [4. Up to now, although ones believe that the hard thermal loop(HTL) in temperature field theory can be got from QGP kinetic theory 2 , 5], it has no a valid scheme for solving the kinetic equations under the condition of non-Abelian gauge symmetry even if for the perturbative dynamics in QGP. We know a standard perturbative approach to the kinetic equations of a plasma is to expand the equations in coupling constant for a gauge theory 2], which doesn't leads to a gauge symmetry to be broken. Actually, another usual method in traditional study of plasma is to expand the equations in powers of weak field strength [ [ 6, 6]. Note that the two methods is in full agreement with each other in electromagnetic plasma 8,9 because of Abelian theory(linear dynamics). Thus the two

*Email address zhengxp@iopp.ccnu.edu.cn 
above-mentioned methods are carried on in past works for QGP. However, they both suffer from the shortcomings to be powerlessly overcome. It is well-known that the first gave only a linear leading order, namely, Abelian-like contribution instead of truly non-Abelian ones, while the latter breaks the non-Abelian gauge covariance as was pointed out in $\operatorname{Ref}[2,0$. If we want to settle the above-mentioned problem in the framework of kinetic theory, the kernel of the problem need to be payed special attention to. We think that the kernel has two aspects: the gauge invariance in perturbation and the treatment of the nonlinearity in the equations because of the nonlinear( non-Abelian ) dynamics in QGP.

In this letter, we will just present a new scheme both satisfying SU(3) gauge symmetry and having solvability on basis of non-Abelian kinetic theory. The scheme has a basic idea: After expanding the kinetic equations in coupling constant $g$, we do the iterative calculations in powers of field strength for purely non-Abelian counterpart. It is just so called 'double perturbation' below. We aim at providing a well-defined prescription to treat a number of color dielectric physics. Here we present the framework of the perturbation theory of the kinetic theory and then give the main results of our analysis. This includes the derivation of a general set of perturbative kinetic equations for non-Abelian plasma and the explanations for the gauge invariance and solvability. An explicit application for the calculations of Landau damping rate in closed-to-equilibrium QGP, which has been computed from HTL in field theory 10, 11, is given as well. We are also able to show that of our results are associated with and beyond HTL approximation possibly. We give a more extensive discussion, and further details on computations in another article 12.

Now we begin with the derivation of a set of equations that describe the dynamics associated with non-Abelian fluctuations. It is sufficient to adopt the semiclassical kinetic theory of QGP for studying the thermal effects. The theory is consist of kinetic equations, which reads 13

$$
\begin{gathered}
p^{\mu} D_{\mu} Q_{ \pm}(\mathbf{p}, x) \pm \frac{g}{2} p^{\mu} \partial_{p}^{\nu}\left\{F_{\mu \nu}(x), Q_{ \pm}(\mathbf{p}, x)\right\}=0, \\
p^{\mu} \tilde{D}_{\mu} G(\mathbf{p}, x)+\frac{g}{2} p^{\mu} \partial_{p}^{\nu}\left\{\tilde{F}_{\mu \nu}(x), G(\mathbf{p}, x)\right\}=0,
\end{gathered}
$$

where the letters with represent the corresponding operators in adjoint representation of $\mathrm{SU}(\mathrm{N})$, the generator denoted by $T_{a}$.

And the background field equation, written as

$$
D^{\mu} F_{\mu \nu}^{a}=j_{\nu}^{a}(x),
$$

where covariant derivative $D^{\mu}=\partial^{\mu}+i g A^{\mu}(x)$, field potential $A^{\mu} \equiv A_{a}^{\mu} \tau^{a}$, and field tensor 
$F^{\mu \nu}=\partial^{\mu} A^{\nu}-\partial^{\nu} A^{\mu}-i g f_{a b c} \tau^{c} A_{a}^{\mu} A_{b}^{\nu}, \tau_{a}$, the generator of SU(N), the color currents including external and induced ones, $j_{\nu}^{a}=j_{\nu}^{\text {ext } a}+j_{\nu}^{\text {ind } a}$.

In principle, a perturbative method is often necessary to solve nonlinear equations. Here we take the close-to-equilibrium situation of a QGP as a case to discuss that. We will note that our method is easily extended to out-of-equilibrium QGP latter. Now we follow the same philosophy in traditional kinetic theory to write the distribution functions $Q_{ \pm}(x, p), G(x, p)$ and fields as 2, 13,

$$
A_{\mu}^{a}=a_{\mu}^{a}, \quad Q_{ \pm}=Q_{ \pm}^{\mathrm{eq}}+\delta Q_{ \pm}, \quad G=G^{\mathrm{eq}}+\delta G
$$

The associated density fluctuations and induced fields are random quantities, satisfying $<\delta Q>=0,<\delta G>=0$ and $<a>=0$, where symbol $<>$ denotes the mean value of a quantity. We define field tensor as $f_{\mu \nu}$ corresponding to $a$.

Thus, we obtain a set of kinetic equations for fluctuations from equations(1) and (2)

$$
\begin{gathered}
p^{\mu} D_{\mu} \delta Q_{ \pm}(\mathbf{p}, x) \pm \frac{g}{2} p^{\mu} \partial_{p}^{\nu}\left\{f_{\mu \nu}(x), Q_{ \pm}(\mathbf{p}, x)\right\}=0, \\
p^{\mu} \tilde{D}_{\mu} G(\mathbf{p}, x)+\frac{g}{2} p^{\mu} \partial_{p}^{\nu}\left\{\tilde{f}_{\mu \nu}(x), G(\mathbf{p}, x)\right\}=0 .
\end{gathered}
$$

According to perturbative dynamics, the latter limitation on the amplitude of the fields ensures in particular the consistency of soft covariant derivative: assuming $a_{\mu} \sim T, i \partial_{\mu} \sim$ $g a_{\mu}$ 14]. We now employ our 'double perturbation' scheme to expand the distribution functions in powers of $g$ in the first step, and then iterate repeatedly the nonlinear parts in expanded equations in field quantity. These can be expressed as

$$
\begin{gathered}
\delta Q=g Q^{(1)}+g^{2} Q^{(2)}+\cdots, \quad \delta G=g G^{(1)}+g^{2} G^{(2)}+\cdots, \\
Q^{(n)}=\sum_{\lambda=1} Q^{(n, \lambda)}, \quad G^{(n)}=\sum_{\lambda=1} G^{(n, \lambda)} .
\end{gathered}
$$

Thus we obtain a series of equations

$$
\begin{gathered}
p^{\mu} \partial_{\mu} Q_{ \pm}^{(n)} \pm i g \sum_{\lambda} p^{\mu}\left[a_{\mu}, Q_{ \pm}^{(n, \lambda)}\right]+\frac{g}{2} p^{\mu}\left\{f_{\mu \nu}, \partial_{p}^{\nu} Q_{ \pm}^{(n-1)}\right\}=0, \\
p^{\mu} \partial_{\mu} G^{(n)}+i g \sum_{\lambda} p^{\mu}\left[\tilde{a}_{\mu}, G^{(n, \lambda)}\right]+\frac{g}{2} p^{\mu}\left\{\tilde{f}_{\mu \nu}, \partial_{p}^{\nu} G^{(n-1)}\right\}=0, \\
D^{\mu}(a) f_{\mu \nu}^{(n) a}=j_{\nu}^{(n) \text { ind } a}(x),
\end{gathered}
$$

where $n, \lambda$ represent respectively the powers of coupling constant and field quantity. The induced currents associated with each order density fluctuations are determined by

$$
j_{\nu}^{(n) \text { ind } a}=g \int \frac{d^{3} p}{(2 \pi)^{3}} \frac{p_{\nu}}{p_{0}} \operatorname{Tr}\left(2 N_{f} \tau^{a}\left[Q_{+}^{(n)}-Q_{-}^{(n)}\right]+2 T^{a} G^{(n)}\right)
$$


where $N_{f}$ account for flavour numbers of quarks, the factors of 2 , the spin degrees of freedom.

Equations (9), (10), (11) and (12) form a basis of perturbation theory of non-Abelian kinetic theory.

The short comments on this follow

1. The non-Abelian gauge symmetry is exactly preserved in each order perturbative equations of (9) and (10) at $g$. As a consequence $Q_{ \pm}^{(n)}$ and $G^{(n)}$, like $Q_{ \pm}$and $G$, transform separately as SU(3) gauge-invariant scalars. Because the summations of non-Abelian terms in (9) and (10) can be done infinitely, the 'double perturbation' method we use guarantees that the result of a physical quantity is also gauge-invariant within any high precision (because we can take $\lambda$ as any large number).

2. In general, it is a difficult task to straightforwardly solve the equations (5) and (6) due to the the nonlinearity of non-Abelian counterparts 15 . However, the summations over $\lambda$ in equations (9) and (10) imply that the iterative evaluations in the powers of field quantity ensure the solvability of non-Abelian counterparts. It is known that the perturbative expansions only in field quantity, which had been carried out in the past works 证, 6, 7, have to suffer from disadvantage of breaking non-Abelian gauge symmetry of a theory at each step of approximation calculation, as was pointed out in Ref [16, because the all higher orders in field quantity contain the contributions to the relevant lower order in coupling constant $g$ for the density fluctuations. So the problem of resummation of all same order contributions in $g$ shall appear to get a gauge-independent physical result in the past works [6, 7, 16. While our approach automatically singles out all contributions of the same order in $g$ together. In this sense, the treatment with non-Abelian contributions in our approach may give the theoretical basis of the resummation technique.

3. It can be easily verified that not only total color current but also each order current are covariant conservation from (12),

$$
D^{\mu} j_{\mu}^{(n) \text { ind } a}=0 .
$$

This is automatically consistent with field equation (11).

4. Our theory will be easily generalized to out-of-equilibrium situations so long as the distribution functions $Q_{ \pm}(x, p), G(x, p)$ and fields $A_{\mu}$ are decomposed into regular terms and density fluctuations, namely, the expression (4) is replaced by

$$
A_{\mu}^{a}=<A_{\mu}^{a}>+a_{\mu}^{a}, \quad Q_{ \pm}=<Q_{ \pm}>+\delta Q_{ \pm}, \quad G=<G>+\delta G .
$$

We had made use of the division in the study of QGP 迎, so do it in other works [7, 15, 16] and in particular, the gauge consistent of the decomposition had been proved in $\sqrt{15}$. Here 
we can only put forward a gauge-consistent fluctuation dynamics, while Litim et al think that the regular parts describe mean field dynamics 15, in full accordance with the related works 17.

5. One will have a more penetrating understanding of those from calculations latter.

In the close to equilibrium situation as an example, we now apply the theory to calculate gloun damping rate for a purely gluonic gas. Then we write out the first order equation in coupling constant

$$
\begin{gathered}
p^{\mu} \partial_{\mu} G^{(1)}+i g \sum_{\lambda} p^{\mu}\left[\tilde{a}_{\mu}, G^{(1, \lambda)}\right]+g p^{\mu} \tilde{f}_{\mu \nu} \partial_{p}^{\nu} G^{(0)}=0 \\
D^{\mu}(a) f_{\mu \nu}^{(1) a}=j_{\nu}^{(1) \operatorname{ind} a}(x),
\end{gathered}
$$

Marking the summation term by $\tilde{S}$, we give

$$
\begin{aligned}
\tilde{S}= & -\int \frac{d^{4} k}{2 \pi)^{4}} \partial_{p}^{\nu} G^{(0)}\left(i g \int \frac{d^{4} k_{1}}{(2 \pi)^{4}} \frac{d^{4} k_{2}}{(2 \pi)^{4}} \delta\left(k-k_{1}-k_{2}\right) \frac{1}{p \cdot k_{2}}\left[p \cdot \tilde{a}\left(k_{1}\right), p^{\mu} \tilde{f}_{\mu \nu}\left(k_{2}\right)\right]\right. \\
& +i g^{2} \int \frac{d^{4} k_{1}}{(2 \pi)^{4}} \frac{d^{4} k_{2}}{(2 \pi)^{4}} \frac{d^{4} k_{3}}{(2 \pi)^{4}} \delta\left(k-k_{1}-k_{2}-k_{3}\right) \frac{1}{p \cdot\left(k_{2}+k_{3}\right) p \cdot k_{2}}\left[p \cdot \tilde{a}\left(k_{1}\right),\left[p \cdot \tilde{a}\left(k_{2}\right), p^{\mu} \tilde{f}_{\mu \nu}\left(k_{2}\right)\right]\right] \\
& +\cdots \cdots)
\end{aligned}
$$

Therefore we can give a general formula of $G^{(1)}(\omega, \mathbf{k})$ from equation(15) in momentum space.

We know that the current induced by fluctuations can be expressed as

$$
\left.j_{\nu}^{(1) \text { ind } a}=g \int \frac{\mathrm{d}^{3} \mathrm{p}}{(2 \pi)^{3}} \frac{p_{\nu}}{p_{0}} \operatorname{Tr}\left[2 T_{a} G^{(1)}(\mathbf{p}, x)\right]\right\},
$$

Following the same method in [4], we can derive response equation of medium from kinetic and field equations. In the approximation here $\lambda$ remains to be 2 ,for an example at zero momentum, we get from field equation (16) together with equations (15) and (18),

$$
\begin{aligned}
& -\omega^{2} \varepsilon^{(\sigma)}(\omega, 0)<a^{2}(\omega, 0)> \\
& =\quad N g^{2} \int \frac{d \omega_{1} d^{3} k_{1}}{(2 \pi)^{4}} d \omega_{2} \delta\left(\omega-\omega_{1}-\omega_{2}\right) \frac{1}{\omega^{2} \varepsilon^{(\sigma)}} \\
& \times \quad\left(\kappa_{l l}<a_{l}^{2}\left(\omega_{1}, \mathbf{k}_{\mathbf{1}}\right)><a_{l}^{2}\left(\omega_{2}, \mathbf{k}_{\mathbf{1}}\right)>+\kappa_{t l}<a_{t}^{2}\left(\omega_{1}, \mathbf{k}_{\mathbf{1}}\right)><a_{l}^{2}\left(\omega_{2}, \mathbf{k}_{\mathbf{1}}\right)>+\kappa_{t t}<a_{t}^{2}\left(\omega_{1}, \mathbf{k}_{\mathbf{1}}\right)><a_{t}^{2}\left(\omega_{2}, \mathbf{k}_{\mathbf{1}}\right)>\right) \\
& +\quad N g^{2} \int \frac{d^{4} k_{1}}{(2 \pi)^{4}}\left(<\mathbf{a}^{2}\left(k_{1}\right)-<a^{2}\left(k_{1}\right)>\right)<a^{2}(\omega, 0)> \\
& +\quad N g^{2} m_{g}^{2} \int \frac{d^{4} k_{1}}{(2 \pi)^{4}} d \mathbf{v} \frac{1}{\omega} \frac{1}{v \cdot\left(k_{1}-k\right)}\left(\frac{\omega_{1}}{v \cdot k_{1}}-1\right)<(\mathbf{v} \cdot \mathbf{a})^{2}\left(k_{1}\right)<(\mathbf{v} \cdot \mathbf{a})^{2}(\omega, 0)>=0
\end{aligned}
$$

where $\varepsilon^{(\sigma)}$ denotes dielectric function for $\sigma$ mode, $\sigma$ taking longitudinal wave $l$ or transverse one $t, \kappa_{l l}, \kappa_{t l}, \kappa_{t t}$ are coefficient functions of $\omega_{1}, \omega_{2}, \mathbf{k}_{1}$, associated with the definite interactions of plasmons.

We know that the damping is connected with the imaginary parts in (19). Obviously, the second term of the right-hand side has no imaginary part, then the damping will originate 
from the physical processes described by the first and third terms of the right-hand side. Therefore we give the Landau damping rate with ease

$$
\gamma^{(\sigma)}(0)=\left(a^{s}+a^{c}\right) \frac{N g^{2} T}{24 \pi}
$$

with

$$
\begin{gathered}
a^{s}=6 \int \mathbf{k}_{1}^{2} d k_{1} d \omega_{1} d \omega_{2} \delta\left(m_{g}-\omega_{1}-\omega_{2}\right)\left(K_{l l} \rho_{l} \rho_{l}+K_{t l} \rho_{t} \rho_{l}+K_{t t} \rho_{t} \rho_{t}\right) \\
a^{c}=24 \pi \int k_{1}^{2} d k_{1} d \omega_{1}\left(\frac{\left(\omega_{1}-m_{g}\right)^{3}}{m_{g} k_{1} \omega_{1}^{4}} \rho_{l}\left(k_{1}\right)-\frac{\omega_{1}-m_{g}}{m_{g} k_{1} \omega_{1}}\left(1-\frac{\left(\omega_{1}-m_{g}\right)^{2}}{k_{1}^{2}}\right) \rho_{t}\left(k_{1}\right)\right) .
\end{gathered}
$$

where $K_{l l}=\frac{\mathbf{k}_{1}^{4}}{\omega_{1}^{3} \omega_{2}^{3}} \kappa_{l l}, K_{t l}=-\frac{\mathbf{k}_{1}^{2}}{\omega_{1} \omega_{2}^{3}} \kappa_{t l}, K_{t t}=\frac{1}{\omega_{1} \omega_{2}} \kappa_{t t}, \rho_{l}, \rho_{t}$ represent longitudinal and transverse spectral densities.

The forms of $a^{s}$ and $a^{c}$ show that two typical physical processes are contributed to Landau damping. The former describes the self-coupling interaction of a mode due to a constraint of the $\delta$ function, which has a good formal correspondence to the result of HTL in field theory [1], the latter represents the collisional interaction of two plasmons or quasiparticles, which describes the long-range interaction in QGP. The numerical result of $a^{c}$ has been arrived at 5.973, while the estimation of $a^{s}$ is of well-matched order with $a^{c}$.

In conclusion, we have presented a 'double perturbation' idea and given the derivation of a series of perturbative equations. It means that our approach give a new and deeper perspective to perturbation theory and thereby settle a methodological problem in the study of a non-Abelian kinetic theory.

The study of the dielectric physics in QGP will be advanced by applying this new theory. This includes to get a non-Abelian gauge-consistent dielectric tensor and discuss the responses of a QGP to external sources 1 , 18, and so on. We must emphatically point out that since our approach has led $\lambda$-point functions (or correlators) into first-order fluctuations in coupling constant $g$, the physics beyond a equilibrium state will play more vital role in a QGP than a electromagnetic plasma [4, 7].

We have initially studied the Landau damping with this approach as a case at zero momentum in the last part of our analysis. Consequently, both self-coupling interaction of a mode and the interaction of two plasmons contribute to the damping rate. The physical mechanism for two-plasmon interaction can clearly be expressed as a two-body 'collision' from long-range interaction of two quasiparticles. We need to make it clear further as for the self-coupling interaction, which has showed ,in form, the similar 2-2 scattering and 2-3 scattering processes contained in the self-energy of HTL[1].

We also believe that this approach will provide more and better results to uncover the deeper link between kinetic theory and field theory searched for by many investigators [2, 19]. 
This work is supported by the National Nature Science Fund of China.

\section{References}

[1] U.Heinz,Phys.Rev.Lett.51,351(1983);56, 93(c)(1986);Ann.Phys.(N.Y.)161,48(1985);168,148(1986);

H.-Th.Elze, M.Gyulassy and D.Vasak,Phys.Lett. B177,402(1986);Nucl.Phys.B276,706(1986); H.-Th. Elze, Z.Phys. C38,211(1988);

S.Mrówczyński, Phys.Rev. D39,1940(1989);J.-P.Blaizot and E.Iancu, Nucl.Phys.B390,589(1993);Phys.Rev.Lett. 72,3317(1994);Phys.Lett.B326,138(1994);

D.F.Litim and C.Manuel, hep-ph/9906210.

[2] P.F.Kelly, Q.Liu,C C.Lucchesi and C.Manuel, Phys.Rev.Lett. 72,3461(1994); D50,4209(1994).

[3] D.J.Gross, R.D.Pisarski and L.G.Yaff, Rev.Mod.Phys. 53,43(1981);R.D.Pisarski, Phys. Rev.Lett.63,1129(1989);

E.Braaten and R.D.Pisarski, Nucl.Phys. B337,569(1990);J.Frenkel and J.C.Taylor, Nucl.Phys. B334,199(1990).

[4] Zheng Xiaoping, Li Jiarong, Phys. Lett B409,45(1997); Nucl. Phys. A639,705(1998).

[5] J.-P.Blaizot and E.Iancu, Phys.Rev.Lett. 70,3376(1993).

[6] Zhang Xiaofei and Li Jiarong, Phys. Rev.C52,964(1995);J.Phys.G21,1483(1995).

[7] Yu.A.Markov and M.A.Markova, hep-ph/9902397, to published by Transp.Theor.Stat.Phys.

[8] V.N.Tsytovich, Theory of turbulent plasma, Plenum,New.York,1977.

[9] A.G.Sitenko, Fluctuations and Non-linear Wave interactions in plasma, Pergamon, Oxford, 1990.

[10] E.Braaten,R.Pisarski, Pyhs.Rev.Lett. 64,1338(1990).

[11] E.Braaten,R.Pisarski, Phys.Rev.D42,R2156(1990).

[12] Zheng Xiaoping, Chen Jisheng and Li Jiarong, in preparation.

[13] H.-Th. Elze and U.Heinz,Phys.Rep. 183,81(1989). 
[14] J.-P.Blaizot and E.Iancu, Nucl. Phys.B421,565(1994);434,662(1995).

[15] D.F.Litim and C.Manuel, Phys.Rev.Lett. 82, 4991(1999).

[16] Yu.A.Markov and M.A.Markova, hep-ph/9909505.

[17] D.Bödeker, Phys.Lett. B426,351(1998).

[18] S.Mrówczyński, Phys. Lett. B269,383(1991);Yuji Koike and T.Matsui, Phys.Rev.D45,3237(1992).

[19] R.D.Pisarski, hep-ph/9710371. 